\title{
Влияние барьерных контактов на транспорт носителей заряда в однородных структурах из GaAs, легированных глубокими центрами $\mathrm{Cr}$ и EL2
}

\author{
(C) М.Г. Верхолетов, И.А. Прудаев \\ Томский государственный университет, \\ 634050 Томск, Россия \\ E-mail: verkhmaks@yandex.ru
}

Поступила в Редакцию 6 апреля 2021 г.

В окончательной редакции 15 апреля 2021 r.

Принята к публикации 15 апреля 2021 г.

Представлены результаты исследования транспорта носителей заряда в структурах из GaAs для детекторов ионизирующих излучений и сверхбыстрых фотоэлектрических переключателей, содержащих глубокие донорные EL2 центры и акцепторные уровни Cr. Исследованы структуры в трех конфигурациях: $p-i-n$-, $n-i-n$ - и $p-i-p$-типов. Решалась система дифференциальных уравнений для температуры носителей заряда, уравнений Пуассона и непрерывности с использованием коммерческого пакета проектирования. Установлено, что выбор типа барьерного слоя позволяет контролировать однородность напряженности электрического поля в структурах. Показано, что наилучшей однородностью напряженности поля обладают структуры $p-i-p$-типа.

Ключевые слова: детекторы ионизирующего излучения, фотоэлектрические коммутаторы, арсенид галлия, глубокие уровни, транспорт носителей заряда.

DOI: 10.21883/FTP.2021.08.51142.9659

\section{1. Введение}

Структуры из GaAs c однородным легированием глубокими центрами применяются для изготовления различных приборов. На сегодняшний день наибольший практический интерес вызывают детекторы ионизирующих излучений и заряженных частиц [1-3], а также сверхбыстрые фотоэлектрические переключатели (HG PCSS - high-gain photoconductive semiconductor switch) [4-6]. Введение глубоких центров в GaAs приводит к росту удельного сопротивления материала при выполнении некоторых статистических условий [1]. Это позволяет добиться низких темновых токов и высоких напряжений лавинного пробоя. На практике широкое распространение получили структуры с глубокими донорными центрами EL2 (антиструктурный дефект $\mathrm{As}_{\mathrm{Ga}}$ ) или глубокими акцепторными центрами Cr. Энергетические уровни данных центров расположены близко к середине запрещенной зоны GaAs, что позволяет создавать материал с предельно высоким удельным сопротивлением $\rho=10^{7}-10^{9} \mathrm{OM} \cdot$ см.

Барьерные слои в приконтактных областях упомянутых детекторов и переключателей играют важную роль в процессе токопереноса и непосредственно влияют на их эффективность [2,3,6-10]. Эффективность этих структур во многом определяется как однородностью распределения электрического поля в них в рабочем режиме, так и величиной темнового тока, протекающего в структуре. Ранее установлено, что увеличение толщины активной области при условии сохранения однородности электрического поля позволяет увеличить эффективность регистрации детекторов $[2,3,7]$ и напря- жение переключения HG PCSS [5,6]. Для HG PCSS c более однородным распределением поля наблюдается больший ресурс по количеству переключений [6]. Темновые токи также снижаются при увеличении толщины данных приборов. Кроме того, для снижения темновых токов и увеличения напряжения пробоя предпринимаются попытки использования блокирующих барьерных слоев в приконтактных областях [2,6-10].

Известно, что в структурах с глубокими донорными EL2 центрами в неравновесных условиях захват электронов на глубокие центры приводит к сильной неоднородности поля вблизи барьерного „катодного“ слоя $[2,12]$. В структурах, компенсированных глубоким акцептором $\mathrm{Cr}$, инжектирующий барьерный слой, в зависимости от степени компенсации структуры, также определяет однородность распределения электрического поля и, как следствие, нелинейность вольт-амперной характеристики (BAX) [13].

Ранее не проводилось исследование влияния разного типа барьерных слоев на однородность распределения поля в структурах из GaAs с глубокими центрами EL2 и Cr. B настоящей работе будет показано, как однородность электрического поля зависит от типа проводимости барьерных слоев, и как эти слои влияют на линейность BAX структур из GaAs при наличии глубоких акцепторных уровней хрома и EL2 центров.

\section{2. Методика расчета}

В данной работе путем численного моделирования решалась одномерная задача для структур $p-i-n-, n-i-n-$ 
и $p-i-p$-типов на основе GaAs, легированного примесями, создающими глубокие донорные и акцепторные энергетические уровни: EL2 и Cr. B расчете геометрические параметры всех структур оставались постоянными: толщина $\mathrm{GaAs}$ составляла 600 мкм, площадь структур $10^{-6} \mathrm{~cm}^{2}$. Катод и анод для всех структур формировались диффузионными профилями; для моделирования $n$-слоя использовался Те, для $p$-слоя - $\mathrm{Zn}$ (максимальные концентрации на поверхности равнялись $\left.2 \cdot 10^{18} \mathrm{~cm}^{-3}\right)$. Отношение концентрации глубоких донорных и акцепторных примесей составляет $N_{d} / N_{a}=0.1[1-3]$.

Расчет проводился путем решения системы дифференциальных уравнений Пуассона для температуры носителей заряда и непрерывности в автоматизированном пакете технологического проектирования Synopsys (TCAD). Плотность тока для электронов и дырок задавалась гидродинамической моделью, описываемой выражениями:

$$
\begin{aligned}
& \mathbf{J}_{n}=\mu_{n}\left(n \nabla E_{C}+k T_{n} \nabla n+f_{n}^{t d} k n \nabla T_{n}\right), \\
& \mathbf{J}_{p}=\mu_{p}\left(p \nabla E_{V}-k T_{p} \nabla p+f_{p}^{t d} k p \nabla T_{p}\right),
\end{aligned}
$$

где $\mu_{n, p}$ - подвижности электронов и дырок, $n$ и $p-$ концентрации электронов и дырок, $E_{C}-$ энергия дна зоны проводимости, $E_{V}-$ энергия потолка валентной зоны, $k$ - постоянная Больцмана, $f_{n, p}^{t d}-$ константы термодиффузии, $T_{n, p}$ - температура электронов и дырок.

В расчетах учтены модели перезарядки глубоких уровней, неполной ионизации примесей и эффекта лавинного умножения $[13,14]$. Степень заполнения глубоких уровней определяется темпами захвата и эмиссии носителей заряда и описывается уравнениями [15]

$$
\begin{aligned}
f^{n} & =\frac{\Sigma c_{i}^{n}}{\Sigma\left(c_{i}^{n}+e_{i}^{n}\right)}, \\
f^{p} & =\frac{\Sigma e_{i}^{p}}{\Sigma\left(e_{i}^{p}+c_{i}^{p}\right)},
\end{aligned}
$$

где $c_{i}^{n, p}-$ скорость захвата носителей заряда из валентной зоны и из зоны проводимости, $e_{i}^{n, p}-$ скорость эмиссии носителей в валентную зону и в зону проводимости.

Аналогично работам $[11,13]$ не использовалась встроенная модель рекомбинации и генерации Шокли-Рида: процессы генерации и рекомбинации свободных носителей заряда рассчитывались непосредственно с учетом захвата и эмиссии с участием двух глубоких центров.

Известно, что в структурах с EL2 центрами наблюдается два характерных эффекта, которые следует учитывать в моделировании $[2,17]$. Они связаны с особенностями темпов захвата и эмиссии носителей. Первый эффект заключается в том, что темп эмиссии электронов оказывается относительно высоким и постоянным вплоть до величин электрического поля $>10^{5} \mathrm{~B} / \mathrm{cm}$ [2]. При комнатной температуре значение темпа эмиссии электронов составляет $e_{C}^{n}=0.045 \mathrm{c}^{-1}$. Второй эффект заключается в том, что сечение захвата электронов для EL2 центров имеет сильную полевую зависимость: при достижении напряженности поля $10^{4} \mathrm{~B} / \mathrm{cm}$, сечение захвата резко возрастает, достигая значения 5.8-30 $10^{-14} \mathrm{~cm}^{2}[2,17]$, что на 2-3 порядка превышает исходное (в слабом электрическом поле). Такое увеличение сечения захвата способствует нейтрализации EL2 центров, так как скорость захвата электронов также резко возрастает. В настоящей работе зависимость сечения захвата EL2 центров от напряженности электрического поля задавалась соотношением:

$$
\begin{gathered}
\sigma_{n}^{e n h}=\sigma_{n}^{0}\left[\frac{1}{2}+\frac{[1+(\alpha-1) \exp (\alpha)]}{\alpha^{2}}\right], \\
\alpha=\frac{1}{k T} \sqrt{\frac{q^{3} F}{\pi \varepsilon_{p f}}}
\end{gathered}
$$

где $\sigma_{n}^{0}-$ сечение захвата при нулевом значении напряженности электрического поля, $F-$ напряженность электрического поля, $\varepsilon_{p f}$ - произведение электрической постоянной на относительную диэлектрическую проницаемость (подгоночный параметр).

На рис. 1, а представлены полевые зависимости сечения захвата электронов для EL2 центров, используемые в настоящей работе в сравнении с экспериментальными данными из литературы [3,18-20]. Моделирование на тестовых структурах, легированных EL2 центрами, показало, что используемая полевая зависимость (5), (6) позволяет корректно описать экспериментальные данные, представленные в литературе: при увеличении поданного напряжения до некоторого порогового значения, за счет интенсивного захвата электронов EL2 центрами, в области катода формируется домен электрического поля с напряженностью $\sim 10^{4} \mathrm{~B} / \mathrm{cM}$. Дальнейшее увеличение приложенного напряжения приводит к расширению сформировавшегося домена со скоростью $\sim 1$ мкм/В (рис. $1, b)$, что хорошо согласуется с результатами других расчетов и экспериментов [2,3,18-20].

Энергия ионизации EL2 центров задавалась равной 0.75 эB, сечения захвата EL2 центров при равновесных условиях составляют $5 \cdot 10^{-16}$ и $2 \cdot 10^{-18} \mathrm{~cm}^{2}$ для электронов и дырок соответственно. Для глубокого акцептора хрома в эксперименте не наблюдается полевой зависимости сечения захвата. Сечения захвата электронов и дырок глубокого акцептора хрома задавались равными $3 \cdot 10^{-19}$ и $8 \cdot 10^{-17} \mathrm{~cm}^{2}$, энергия ионизации хрома 0.79 эВ.

При моделировании статических характеристик структур с отрицательной дифференциальной подвижностью, таких как GaAs, необходимо учитывать возможное появление нестационарного решения из-за изменения подвижности в результате междолинного перехода электронов. В эксперименте нестационарное поведение ВАХ (осцилляции тока) в структурах с $\mathrm{Cr}$ не наблюдается [1-3], что может быть связано с некоторыми особенностями рассеяния носителей заряда в данном материале. В связи с этим в моделировании для предотвращения появления нестационарных решений использовалась 

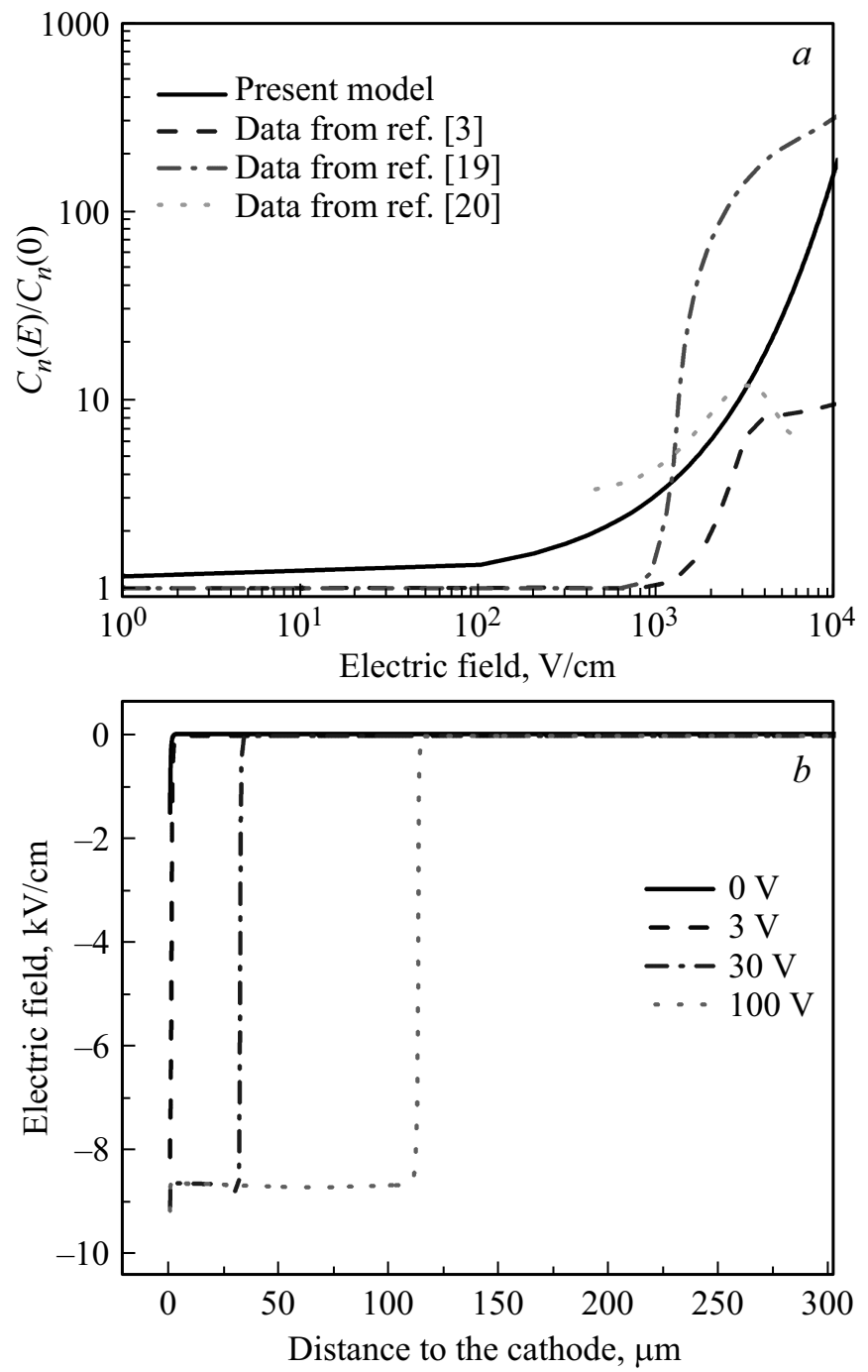

Рис. 1. Зависимость сечения захвата от напряженности электрического поля для EL2 центров, используемая в данной работе и другими авторами $(a)$, рассчитанное распределение электрического поля в объеме структуры, легированной EL2 центрами, при различном поданном напряжении $(b)$.

упрощенная модель подвижности для электронов и дырок, которая описывается уравнением:

$$
\mu(F)=\frac{\mu_{\mathrm{low}}}{\left[1+\left(\frac{\mu_{\mathrm{low}} F_{h f s}}{v_{\mathrm{sat}}}\right)^{\beta}\right]^{1 / \beta}},
$$

где $\mu_{\text {low }}$ - подвижность в слабом электрическом поле, $v_{\text {sat }}$ - дрейфовая скорость, $F_{h f s}-$ напряженность электрического поля, $\beta-$ коэффициент, зависимый от температуры.

Тестовые расчеты показали, что использование модели отрицательной дифференциальной подвижности электронов для структур из $\mathrm{GaAs}$, легированных $\mathrm{Cr}$ и EL2, приводят к расходимости решения при достижении напряженности поля, близкой к $3-4 \cdot 10^{3} \mathrm{~B} / \mathrm{cm}$.

\section{3. Результаты и их обсуждение}

На рис. 2 представлены рассчитанные вольт-амперные характеристики исследуемых структур. Из рис. 2 видно, что BAX структур имеют качественные различия. Рассмотрим далее механизмы формирования ВАХ для каждой структуры в отдельности.

Рассмотрим структуру $n-i-n$-типа, которая имеет наиболее ярко выраженные нелинейные участки. Для более удобного описания разобьем ВАХ структуры на четыре участка. Первая область линейного роста тока описывается законом Ома $I \propto U$ : увеличение напряжения от 0.1 до $1 \mathrm{~B}$ приводит к увеличению тока в 10 раз. При анализе распределения напряженности поля и концентрации носителей заряда установлено, что концентрация электронов и дырок остается практически неизменной для данного участка ВАХ (уменьшается на $1 \%$ ), а напряженность поля при этом возрастает в 10 раз.

На втором участке BAX падение напряжения происходит на обратно-смещенном $i-n$-переходе, так как его сопротивление становится много больше сопротивления объема $i$-слоя и прямосмещенного $i-n$-перехода. Ток, протекающий через структуру в диапазоне напряжений от 1 до $10 \mathrm{~B}$, обусловлен генерацией носителей заряда в обратносмещенном $i-n$-переходе, где сильно возрастает напряженность электрического поля (на это указывают результаты, представленные на вставке к рис. 3).

На третьем участке BAX при напряжении от 10 до $100 \mathrm{~B}$ ток резко возрастает. Анализ профилей концентрации носителей заряда показывает, что структура переходит в режим монополярной инжекции (концентра-

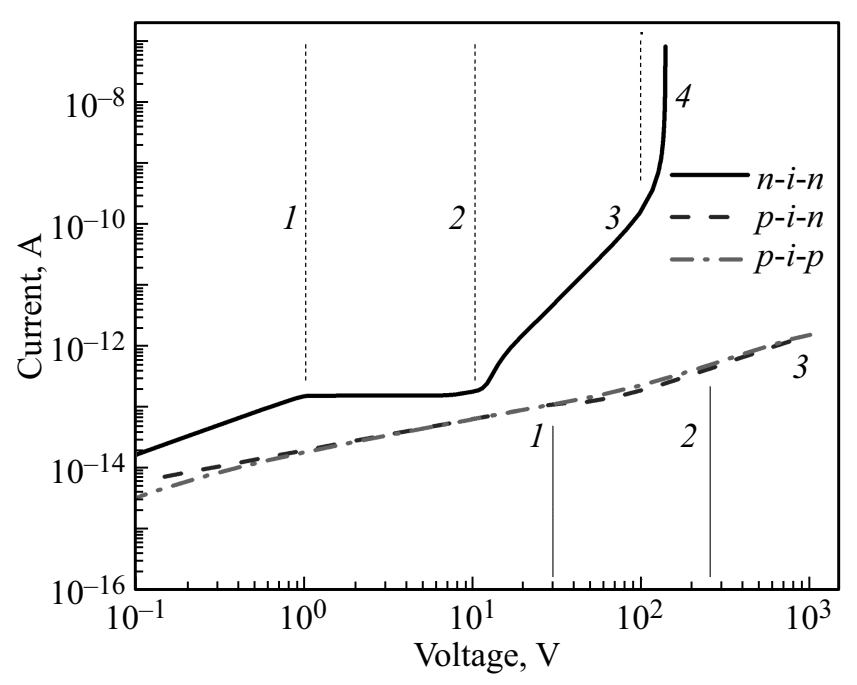

Рис. 2. Вольт-амперные характеристики трех структур с различными барьерными слоями. Штриховыми линиями показано условное разбиение $\mathrm{BAX} n-i-n$-структуры на участки с различными механизмами токопереноса. Сплошными линиями показано условное разбиение $\mathrm{BAX} p-i-n$ - и $p-i-p$-структур (полярность для всех структур: „минус“ - слева, „плюс“ справа). 


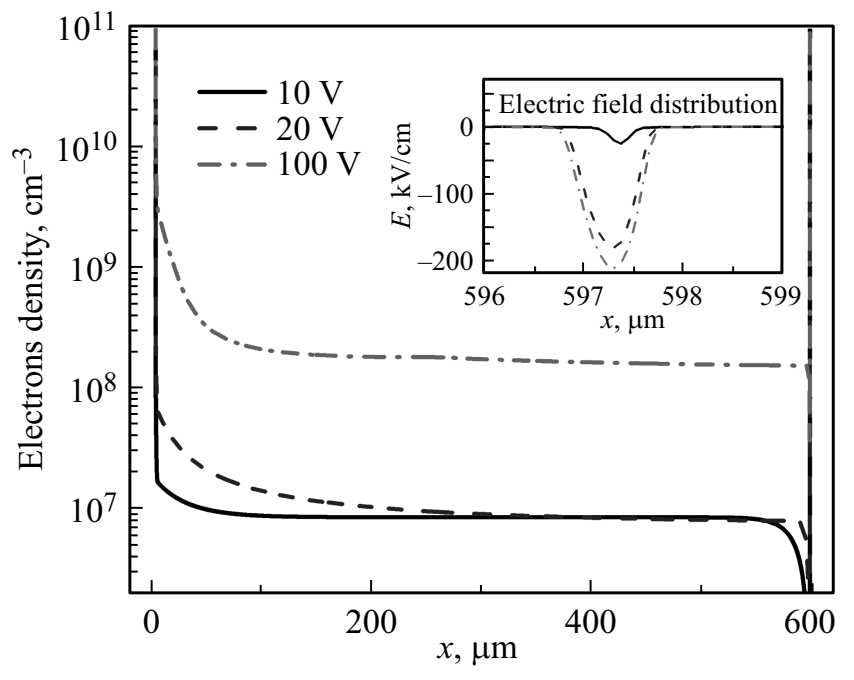

Рис. 3. Распределение электронов в $n-i-n$-структуре в зависимости от величины приложенного внешнего напряжения. На вставке - распределение напряженности электрического поля в области анода $n-i-n$-структуры.

ция электронов более чем на порядок превышает концентрацию дырок). Слева электроны интенсивно инжектируются из катода (рис. 3 ), так как высота $n-i$-барьера снижается.

В это же время в области анода из-за сильного увеличения напряженности электрического поля в области обратносмещенного $i-n$-перехода возникает пробой с сопутствующей генерацией лавинных дырок. При этом темп лавинной генерации возрастает с $1 \cdot 10^{-14}$ до $7 \cdot 10^{-16} \mathrm{~cm}^{-3} \cdot \mathrm{c}^{-1}$ при увеличении напряжения с 10 до 100 В. Лавинные дырки обеспечивают достаточный темп рекомбинации инжектированных электронов в области анода. В результате третий участок ВАХ может быть описан в рамках теории монополярной инжекции электронов в диодах с длинной базой [21], когда сила тока возрастает пропорционально квадрату напряжения $I \propto U^{2}$. Очевидно, что изменение времени жизни носителей заряда или параметров лавинной генерации должно приводить к количественным изменениям на третьем участке BAX (в моделировании данные параметры задаются характеристиками глубоких центров и коэффициентами ударной ионизации).

На четвертом участке BAX происходит резкое увеличение тока, что связано с ростом концентрации электронов, которые переносятся к области лавинного размножения за счет дрейфа. Таким образом, резкий рост тока на четвертом участке вызван положительной обратной связью между инжектирующим $n-i$-переходом и обратносмещенным $i-n$-переходом. Подобный резкий рост тока наблюдался ранее в других структурах на основе GaAs с глубокими уровнями и встречновключенными электронно-дырочными переходами в режиме лавинного пробоя [16].
В структуре $p-i-n$-типа в отличие от предыдущего случая отсутствует барьер, инжектирующий электроны. Анализ распределения электронов (рис. 4) и электрического поля (рис. 5) показывает, что на первом участке, до $30 \mathrm{~B}$, поданное напряжение падает на обратносмещенном $p-i$-переходе.

Обедненная область пространственного заряда (ОПЗ) расширяется по закону $d \propto U^{1 / 2}$, где $d-$ ширина ОПЗ. Это обусловливает слабый рост генерационного тока на данном участке по закону $I \propto U^{1 / 2}$. Важно отметить, что концентрация нескомпенсированных ионов примеси в обедненной области контролируется процессами перезарядки глубоких примесей (3), (4) и составляет крайне малую величину - не более $2 \cdot 10^{11} \mathrm{~cm}^{-3}$. Это обусловливает „быстрое“ расширение ОПЗ на длину всей структуры уже при 30-40 В. Небольшое падение

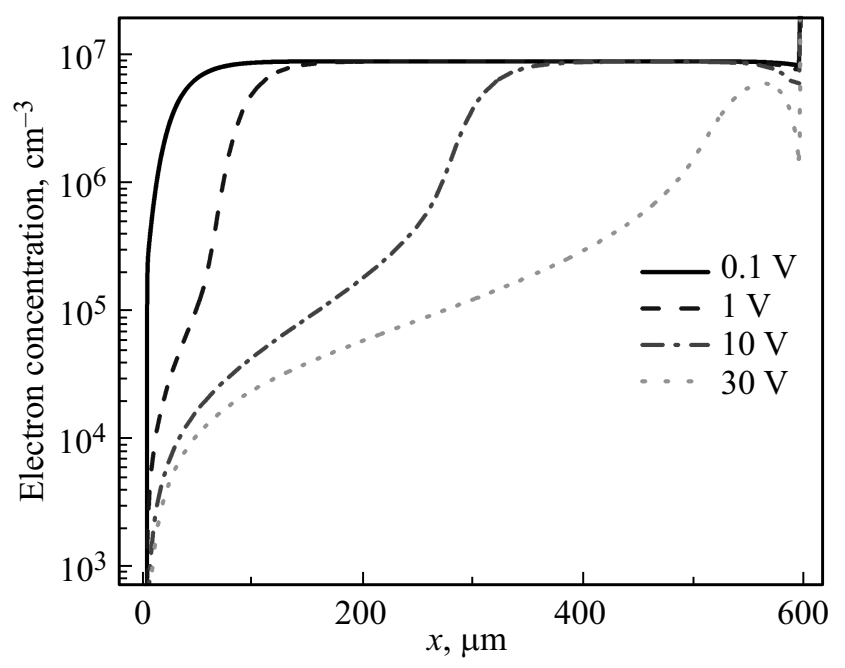

Рис. 4. Распределение электронов в структуре $p-i-n$-типа в зависимости от величины приложенного напряжения.

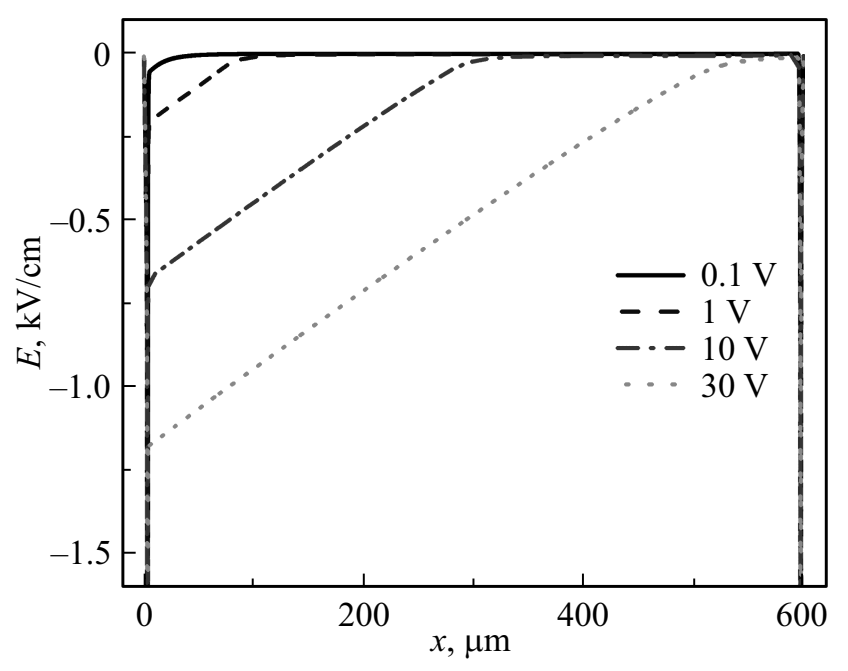

Рис. 5. Распределение напряженности электрического поля в $p-i-n$-структуре при различных значениях поданного напряжения. 
напряжения на обратносмещенном $i-n$-переходе приводит к выходу на насыщение тока в области $U=30-50$ В. Однако дальнейший рост напряжения приводит к росту тока, обусловленному зависимостью подвижности от напряженности поля (7): линейная зависимость $I \propto U$, переходящая к насыщению в области $U>10^{3} \mathrm{~B}$.

BAX структуры $p-i-p$-типа во многом схожа с ВАХ структуры $p-i-n$-типа. Единственным отличием является область напряжений от 30 до $50 \mathrm{~B}$, где ток для последней оказывается ниже на $8 \%$. Данное различие объясняется отсутствием падения напряжения в области анода $p-i-p$-структуры. Стоит отметить, что величина тока в области рабочих напряжений $(U=100-300 \mathrm{~B})$ для $p-i-p$ - и $p-i-n$-структур в 5-10 раз ниже, чем у ранее исследованных структур GaAs с барьерами Шоттки с линейной ВАХ [13]. Очевидно, что детекторы на основе структур $p-i-p$ - и $p-i-n$-типов должны иметь большее соотношение „сигнал/шум“ в сравнении с традиционно используемыми структурами с линейной вольт-амперной характеристикой.

\section{4. Заключение}

Проведенные исследования показали, что выбор типа контактов для структур из GaAs, легированного глубокими донорными (EL2) и акцепторными (Cr) примесями, существенно влияет на механизмы переноса носителей заряда. Установлено, что:

1) в структурах $n-i-n$-типа в результате монополярной инжекции электронов и пробоя обратносмещенного $n-i$-перехода в области $U=10-100 \mathrm{~B}$ на ВАХ формируется участок сверхлинейной степенной зависимости тока от напряжения, что делает данный тип структур непригодным для использования в качестве детекторов ионизирующего излучения и фотоэлектрических переключателей;

2) в структурах $p-i-n$ и $p-i-p$ на участке до $30 \mathrm{~B}$ контролируемая процессами перезарядки концентрация ионов нескомпенсированной примеси составляет крайне малую величину, что обеспечивает „быстрое“ расширение электрического поля на толщину всей структуры. В результате рост тока в этом диапазоне напряжений описывается по закону $I \propto U^{1 / 2}$. Дальнейшее увеличение напряжения приводит к формированию линейного участка ВАХ, обусловленного зависимостью подвижности от напряженности электрического поля.

Таким образом, для изготовления детекторов ионизирующего излучения наиболее предпочтительно использовать структуры $p-i-n$ - и $p-i-p$-типа, так как в области напряжений от 100 до $300 \mathrm{~B}$ они имеют в 5-10 раз меньший ток по сравнению с классически используемыми структурами с барьерами Шоттки. В результате это должно привести к пропорциональному увеличению соотношения „сигнал/шум“ ся одной из основных характеристик детекторов. Также малые токи утечки и большие рабочие напряжения указывают на перспективность применения структур $p-i-n$ - и $p-i-p$-типа в качестве HG PCSS.

\section{Финансирование работы}

Исследование выполнено при финансовой поддержке РФФИ в рамках научного проекта № 20-38-90037.

\section{Конфликт интересов}

Авторы заявляют, что у них нет конфликта интересов.

\section{Список литературы}

[1] A.V. Tyazhev, D.L. Budnitsky, O.B. Koretskay, V.A. Novikov, L.S. Okaevich, A.I. Potapov, O.P. Tolbanov, A.P. Vorobiev. Nucl. Instr. Meth. A, 509, 34 (2003).

[2] M. Rogalla, K. Runge. Nucl. Instr. Meth. A, 434, 44 (1999).

[3] A. Cola, L. Reggiani, L. Vasanelli. Semicond. Sci. Technol., 12, 1358 (1997).

[4] Hanmin Zhao, P. Hadizad, Jung H. Hur, Martin A. Gundersen. J. Appl. Phys., 73, 1807 (1993).

[5] R.P. Joshi, P. Kayasit. J. Appl. Phys., 86, 3833 (1999).

[6] G.M. Loubriel, F.J. Zutavern, A. Mar, M.W. O’Malley, W.D. Helgeson, D.J. Brown, H.P. Hjalmarson, A.G. Baca. 11th IEEE Int. Pulsed Power Conf. (29 June-2 July, 1997, Baltimore, MD, USA).

[7] C.M. Buttar. Nucl. Instr. Meth. Phys. Res. B, 395 (1), 1 (1997).

[8] D.S. McGregor, R.A. Rojeskia, G.F. Knolla, F.L. Terry,jr, J. East, Y. Eisen. Methods Phys. Res. A, 343, 527 (1994).

[9] R. Irsigler, R. Geppert, R. Göppert, J. Ludwig, M. Rogalla, K. Runge, Th. Schmid, M. Webel, C. Weber. Methods Phys. Res. B, 395 (1), 71 (1997).

[10] M. Alietti, C. Canali, A. Castaldini, A. Cavallini, A. Cetronio, C. Chiossi, S. D’Auria, C. del Papa, C. Lanzieri, F. Nava, P. Vanni. Methods Phys. Res. B, 362 (2-3), 344 (1995).

[11] I.A. Prudaev, S.N. Vainshtein, M.G. Verkholetov, V.L. Oleinik, V.V. Kopyev. IEEE Trans. Electron Dev., 68 (1), 57 (2021).

[12] K. Berwick, M. Brozel, C. Butiar, M. Cowperthwaite, Y. Hou. MRS Online. Proc. Library, 302, 363 (1993).

[13] И.А. Прудаев, М.Г. Верхолетов. Письма ЖТФ, 45 (11), 37 (2019).

[14] I.A. Prudaev, V.L. Oleinik, T.E. Smirnova, V.V. Kopyev, M.G. Verkholetov, E.V. Balzovsky, O.P. Tolbanov. IEEE Trans. Electron Dev., 65, 3339 (2018).

[15] Sentaurus Device User Guide. www.sentaurus.dsod.pl/manuals/data/sdevice_ug.pdf

[16] И.А. Прудаев, М.Г. Верхолетов, А.Д. Королёва, О.П. Толбанов. Письма в ЖТФ, 44 (6), 465 (2018).

[17] V.Y. Prinz, S.N. Rechkunov. Phys. Status Solidi B, 118 (1), 159 (1983).

[18] D.S. McGregor, R.A. Rojeski, G.F. Knoll. J. Appl. Phys., 75, 7910 (1994).

[19] L.L. Bonilla, P.J. Hernando, M. Kindelan. Appl. Phys. Lett., 74, 988 (1999).

[20] F. Piazza, P. Christianen, J. Maan. Appl. Phys. Lett., 69, 1909 (1996).

[21] В.И. Гаман. Физика полупроводниковых приборов (Томск, Изд-во Томск. ун-та, 1989) гл. 2, с. 67.

Редактор Г.А. Оганесян 
The influence of Barrier Contacts on Carrier Transport in Homogeneous GaAs Structures Doped with Deep Cr and EL2 Centers

M.G. Verkholetov, I.A. Prudaev

Tomsk State University, 634050 Tomsk, Russia

Abstract The results of studying the transport of charge carriers in GaAs structures doped with deep donor EL2 centers and acceptor levels of $\mathrm{Cr}$ for detectors of ionizing radiation and ultrafast photoelectric switches are presented. Three configurations of structures are investigated: $p-i-n-, n-i-n$ - and $p-i-p$-types. The system of differential equations for the temperature of charge carriers, Poisson's equations and continuity was solved using a commercial software. It was found that the choice of the type of the barrier layer makes it possible to control the uniformity of the electric field strength in the structures. It is shown that $p-i-p$-type structures have the best uniformity of the electric field strength. 